\title{
Expression of miR-221 and miR-489 in breast cancer patients and their relationship with prognosis
}

\author{
FANFAN LI
}

\author{
Department of Breast Surgery, First Teaching Hospital of Tianjin University \\ of Traditional Chinese Medicine, Tianjin 300193, P.R. China
}

Received September 27, 2019; Accepted November 26, 2019

DOI: $10.3892 / \mathrm{ol} .2019 .11196$

\begin{abstract}
Expression of miR-221 and miR-489 in breast cancer patients and their prognostic value were investigated. Sixty-two breast cancer patients admitted to the First Teaching Hospital of Tianjin University of Traditional Chinese Medicine for tumor surgery, from July 2014 to January 2016, were selected as the research group (RG), and 27 female adults who underwent physical examination during the same period were selected as the control group (CG). miR-221 and miR-489 expression levels in the blood samples of the breast cancer patients and the healthy female adults were detected by fluorescence reverse transcription-quantitative PCR (RT-qPCR), and the relationship of the expression levels of miR-221 and miR-489 with the disease prognosis was analyzed. The expression levels of miR-221 and miR-489 in the blood samples of breast cancer patients were $7.13 \pm 1.19$ and $0.88 \pm 0.09$, respectively, and those in the blood samples of healthy individuals were $5.82 \pm 0.84$ and $1.01 \pm 0.12$, respectively. The expression level of miR-221 in the RG was significantly higher than that in the $\mathrm{CG}(\mathrm{P}<0.01)$, while the expression level of miR-489 in the RG was significantly lower than that in the CG $(\mathrm{P}<0.01)$. The area under the curve (AUC) of miR-221 was 0.769, and the AUC of miR-489 was 0.805 . When AUC was equal to 0.88 , the combined detection of the two had higher sensitivity and specificity than the single detection. The 3-year survival rates of miR-221 low-expression group and miR-489 high-expression group were significantly higher than those of the miR-221 high-expression group and miR-489 low-expression group $(\mathrm{P}<0.05)$. miR-221 expression was upregulated and miR-489 expression was downregulated in blood samples of breast cancer patients, which had a certain impact on the patients survival. In the future, miR-221 can
\end{abstract}

Correspondence to: Dr Fanfan Li, Department of Breast Surgery, First Teaching Hospital of Tianjin University of Traditional Chinese Medicine, 314 Anshan West Road, Tianjin 300193, P.R. China E-mail:1hz7xz@163.com

Key words: breast cancer, miR-221, miR-489, prognosis, state of illnesss be used as an effective indicator for diagnosis, treatment and prognosis of breast cancer.

\section{Introduction}

Breast cancer is one of the most common types of tumors among women and the main cause of cancer-related deaths in women worldwide (1). Some studies have revealed that the overall morbidity of breast cancer among Asian women is on the increase (2), and although the breast cancer treatment methods have improved, the death toll from breast cancer has not decreased due to the aging population (3). Due to the high recurrence rate of tumors and the drug resistance to chemotherapy, the efficacy of breast cancer treatments and prognosis of breast cancer have not achieved satisfactory results (4), making breast cancer a hot topic in clinical research. At present, a number of reports (5-7) have proven that microRNAs (miRNAS) are involved in the development of various tumors. miR-221 and miR-489 have been verified to have abnormal expression (8-10) in colorectal cancer and gastric cancer; however, miR-221 and miR-489 expression levels in breast cancer have been rarely reported. Therefore, in the present study it was speculated that miR-221 and miR-489 have also abnormal expression in breast cancer, and experimental analysis was carried out for verification. The aim of the present study was to investigate whether miR-221 and miR-489 can be used as diagnostic indicators and disease prediction indices in breast cancer, and to provide a relevant reference basis and suggestions for the future clinical treatment of breast cancer.

\section{Subjects and methods}

Clinical data. Sixty-two breast cancer patients admitted to the First Teaching Hospital of Tianjin University of Traditional Chinese Medicine (Tianjin, China) for tumor surgery, from July 2014 to January 2016, were selected as the research group (RG), and 27 female adults who underwent physical examination during the same period were selected as the control group (CG). The average age of all subjects was $48.12 \pm 10.36$ years. The study was approved by the Ethics Committee of the First Teaching Hospital of Tianjin University of Traditional Chinese Medicine (Tianjin, China). All subjects who participated in this research had complete clinical data. 
Table I. Primer sequences of miR-221, miR-489 and U6 internal reference gene.

\begin{tabular}{lll}
\hline Genes & \multicolumn{1}{c}{ Upstream primers } & \multicolumn{1}{c}{ Downstream primers } \\
\hline miR-221 & 5'-GGGAAGCTACTAAGTCTGC-3' & 5'-GTGCGTGTCGTGGAGTCG-3' \\
miR-489 & 5'-ACACTCCAGCTGGGGTGACATCACATA-3' & 5'-TGGTGTCGTGGAGTCG-3' \\
U6 & 5'-GCTTCGGCAGCACATATACTAAAAT-3' & 5'-CGCTTCACGAATTTGCGTGTCAT-3'
\end{tabular}

Signed written informed consents were obtained from the participants and/or their guardians.

Inclusion and exclusion criteria. All patients in the RG were diagnosed with breast cancer after biopsy in the Pathology Department of our hospital. The exclusion criteria were as follows: i) Patients with incomplete pathological data; ii) patients with other tumors; iii) patients who had received relevant treatment; iv) patients with other visceral diseases; v) patients with drug allergy history; vi) patient referrals.

Methods. Sixty-two breast cancer patients were selected as the RG and 27 healthy women as the CG. Blood samples were collected from both groups. miR-221 expression (kit purchased from Guangzhou Angfeibio Biotech Co., Ltd.; UTS19324) and miR-489 (kit purchased from Shanghai Yihui Biological Technology Co., Ltd.; HHM0387) were detected by fluorescence reverse transcription-quantitative PCR (RT-qPCR). Total RNA was extracted using TRIzol ${ }^{\circledR}$ kit (Shanghai Mingjing Biology Co., Ltd.; 5003050) according to the manufacturer's protocol. The extracted total RNA was reverse transcribed into cDNA according to the manufacturer's instructions of a reverse transcription kit (Wuhan Chundu Biotech Co., Ltd.; CD-102539GM). Reverse transcription reaction conditions were $50^{\circ} \mathrm{C}$ for $45 \mathrm{~min}$, and the reverse transcriptase was inactivated at $85^{\circ} \mathrm{C}$ for 5 min. cDNA was amplified using an amplification kit (Shanghai Xinyu Biotechnology Co., Ltd.; XY-051021). SYBR Green I (KS26757) was purchased from Shanghai Keshun Biological Technology Co., Ltd. The primer sequences used were synthesized by Thermo Fisher Scientific, Inc. Details are shown in Table I. The following thermocycling conditions were used for qPCR: Pre-denaturation at $94^{\circ} \mathrm{C}$ for $30 \mathrm{sec}$, denaturation at $94^{\circ} \mathrm{C}$ for $5 \mathrm{sec}$, annealing and extension at $60^{\circ} \mathrm{C}$ for $30 \mathrm{sec}$ for 40 cycles. The experiment was conducted 3 times. U6 was used as internal reference and $2^{-\Delta \mathrm{Cq}}$ method was used for quantification (11).

Observation indicators. The expression levels of miR-221 and miR-489 were observed in the two groups, as well as the diagnostic value of miR-221 and miR-489 in breast cancer. The patients in the RG were followed up for 3 years. According to the median values of miR-221 and miR-489 expression levels in the RG, the patients were divided into miR-221 high- and low-expression groups and into miR-489 high- and low-expression groups, respectively. The 3-year survival rates of the high- and low-expression groups were recorded and compared.

Statistical analysis. SPSS 22.0 software (IBM Corp.) was used to statistically analyze the experimental data and generate the relevant figures. The measurement data were expressed as the mean \pm standard deviation. The comparison of the measurement data between two groups was conducted using t-test. Counting data were expressed as percentage [n (\%)], and the comparison of counting data between groups was performed by Chi-square $\left(\chi^{2}\right)$ test. The diagnostic value was analyzed by ROC curve analysis and the survival rates were calculated by Kaplan-Meier method. Log-rank test was used for the comparison of the survival curves. $\mathrm{P}<0.05$ was considered to indicate a statistically significant difference.

\section{Results}

Comparison of clinicopathological characteristics. There was no significant difference in age, weight, family history of breast cancer, marital status, reproductive history, smoking or drinking between the RG and $\mathrm{CG}(\mathrm{P}>0.05)$, proving that the two groups were comparable. Details are presented in Table II.

Comparison of $m i R-221$ and miR-489 expression levels. Blood samples were collected from the RG and CG, and the expression levels of miR-221 and miR-489 were detected. The results revealed that the expression level of miR-221 in the RG was $7.13 \pm 1.19$, significantly higher than that in the CG $(5.82 \pm 0.84)(\mathrm{P}<0.01)$, whereas, the expression level of miR-489 in the RG was $0.88 \pm 0.09$, significantly lower than that in the CG (1.01 \pm 0.12$)(\mathrm{P}<0.01)$ (Fig. 1).

Diagnostic value of $\mathrm{miR}-221$ and $\mathrm{miR}-489$ expression levels in breast cancer. ROC curve analysis revealed that the area under the miR-221 curve was 0.769 , the sensitivity was $61.29 \%$, the specificity was $85.19 \%$, the cut-off value was 6.806 , and the $95 \%$ CI was $66.27-95.81 \%$. The area under the miR-489 curve was 0.805 , the sensitivity was $66.13 \%$, the specificity was $85.19 \%$, the cut-off value was 0.903 , and the $95 \%$ CI was $66.27-95.81 \%$. In addition, the area under the curve of the combined detection of the two was 0.88 , the sensitivity was $64.52 \%$, the specificity was $96.3 \%$, the cut-off value was 0.165 , and the $95 \%$ CI was $81.03-99.91 \%$ (Fig. 2).

Prognosis and survival of patients. According to the median values of the expression levels of miR-221 and miR-489, the patients in the RG were divided into 37 cases of high miR-221 expression ( $\geq 7.13$ ), 22 cases of low miR-221 expression ( $<7.13), 24$ cases of high miR-489 expression $(\geq 0.88)$ and 35 cases of low miR-489 expression (<0.88). By March 2019, 59 patients of the RG were successfully followed up through telephones, hospital re-examination and dropping-in follow-up, with a follow-up success rate of $95.16 \%$. The 1-, 2- and 3-year survival rates in the miR-221 high-expression 
Table II. Comparison of clinicopathological characteristics between the two groups.

\begin{tabular}{|c|c|c|c|c|}
\hline Clinicopathological characteristics & $\begin{array}{l}\text { Research group } \\
\quad(\mathrm{n}=62)\end{array}$ & $\begin{array}{l}\text { Control group } \\
\quad(\mathrm{n}=27)\end{array}$ & $\operatorname{tor} \chi^{2}$ & P-value \\
\hline Age (years) & $41.29 \pm 7.35$ & $39.71 \pm 6.82$ & 0.952 & 0.344 \\
\hline Weight (kg) & $60.47 \pm 7.56$ & $57.42 \pm 6.98$ & 1.790 & 0.077 \\
\hline Family history of breast cancer & & & 1.208 & 0.272 \\
\hline Yes & $21(33.87)$ & $6(22.22)$ & & \\
\hline No & $41(66.13)$ & $21(77.78)$ & & \\
\hline Marital status & & & 1.394 & 0.498 \\
\hline Unmarried & $21(33.87)$ & $9(33.33)$ & & \\
\hline Married & $38(61.29)$ & $18(66.67)$ & & \\
\hline Widowed & $3(4.84)$ & $0(00.00)$ & & \\
\hline Childbearing history & & & 0.048 & 0.826 \\
\hline Having children & $36(58.06)$ & $15(55.56)$ & & \\
\hline Not having children & $26(41.94)$ & $12(44.44)$ & & \\
\hline Smoking & & & 0.185 & 0.667 \\
\hline Yes & $14(22.58)$ & $5(18.52)$ & & \\
\hline No & $48(77.42)$ & $22(81.48)$ & & \\
\hline Drinking & & & 0.203 & 0.653 \\
\hline Yes & $19(30.65)$ & $7(25.93)$ & & \\
\hline No & $43(69.35)$ & $20(74.07)$ & & \\
\hline \multicolumn{5}{|l|}{ Type of carcinoma } \\
\hline Infiltrating carcinoma & $49(79.03)$ & & & \\
\hline Non-infiltrating carcinoma & $13(20.97)$ & & & \\
\hline \multicolumn{5}{|l|}{ Degree of differentiation } \\
\hline Moderately and poorly differentiated & $50(80.65)$ & & & \\
\hline Highly differentiated & $12(19.35)$ & & & \\
\hline \multicolumn{5}{|l|}{ Pathological staging } \\
\hline Stages I-II & $27(43.55)$ & & & \\
\hline Stages III-IV & $35(56.45)$ & & & \\
\hline \multicolumn{5}{|l|}{ Distant metastasis } \\
\hline Yes & $25(40.32)$ & & & \\
\hline No & $37(59.68)$ & & & \\
\hline
\end{tabular}

A

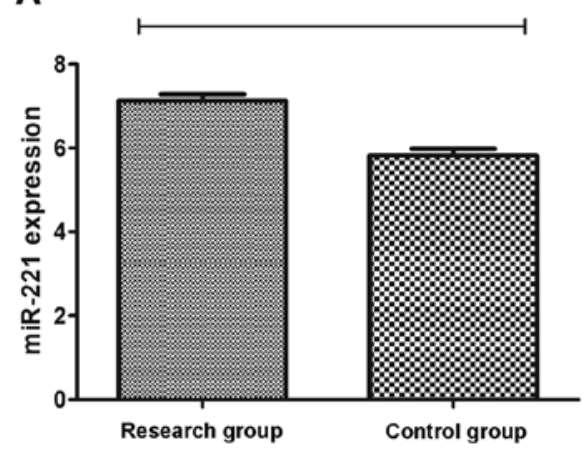

B

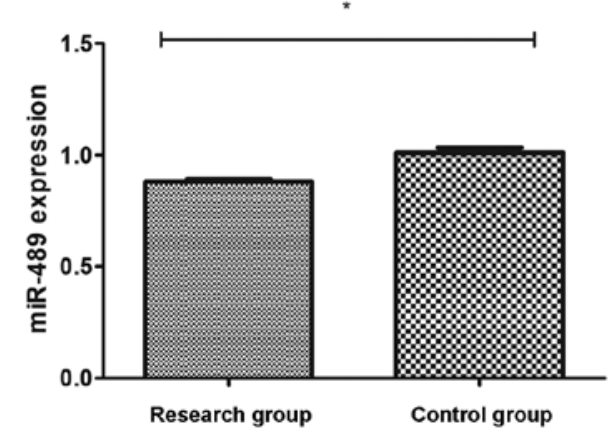

Figure 1. Comparison of the expression levels of miR-221 and miR-489 in the blood samples of patients of the RG and healthy individuals of the CG. (A) The expression level of miR-221 in the RG was significantly higher than that in the CG. (B) The expression level of miR-489 in the RG was significantly lower than that in the CG. ${ }^{*} \mathrm{P}<0.01$. RG, research group; $\mathrm{CG}$, control group.

group were $91.89,78.38$ and $56.76 \%$, respectively, whereas those in the miR-221 low-expression group were 95.45 ,
90.91 and $81.82 \%$, respectively, which were significantly higher than those in the miR-221 high-expression group 
A

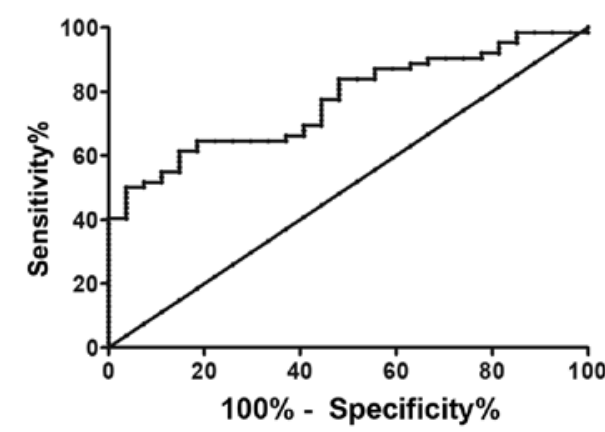

B

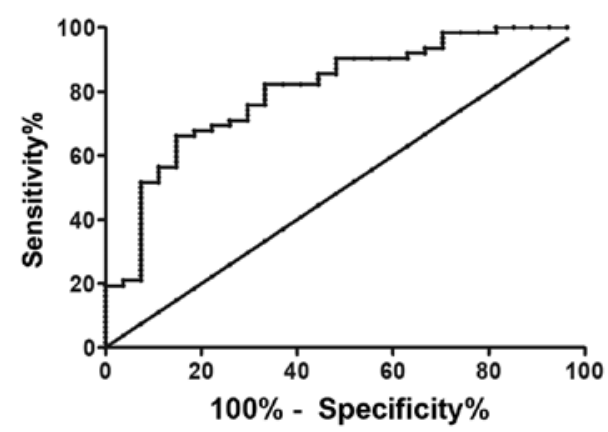

C

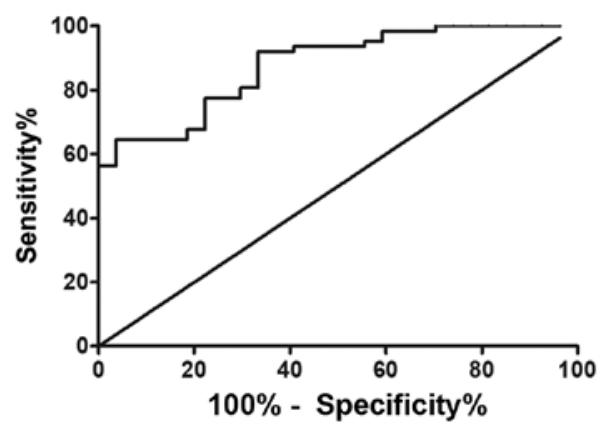

Figure 2. (A) Early diagnostic value of miR-221 in breast cancer (AUC=0.769). (B) Early diagnostic value of miR-489 in breast cancer (AUC=0.805). (C) Early diagnostic value of the combined detection of miR-221 and miR-489 in breast cancer. RG was taken as the independent variable and binary logistic analysis was carried out to obtain the combined detection model: $\log (\mathrm{P})=-4.547+(1.212) \mathrm{x}$ miR-221 $+12.368 \mathrm{x}$ miR-489. When AUC was equal to 0.88 , the model had higher specificity than miR-221 and miR-489 alone. AUC, area under the curve; RG, research group.
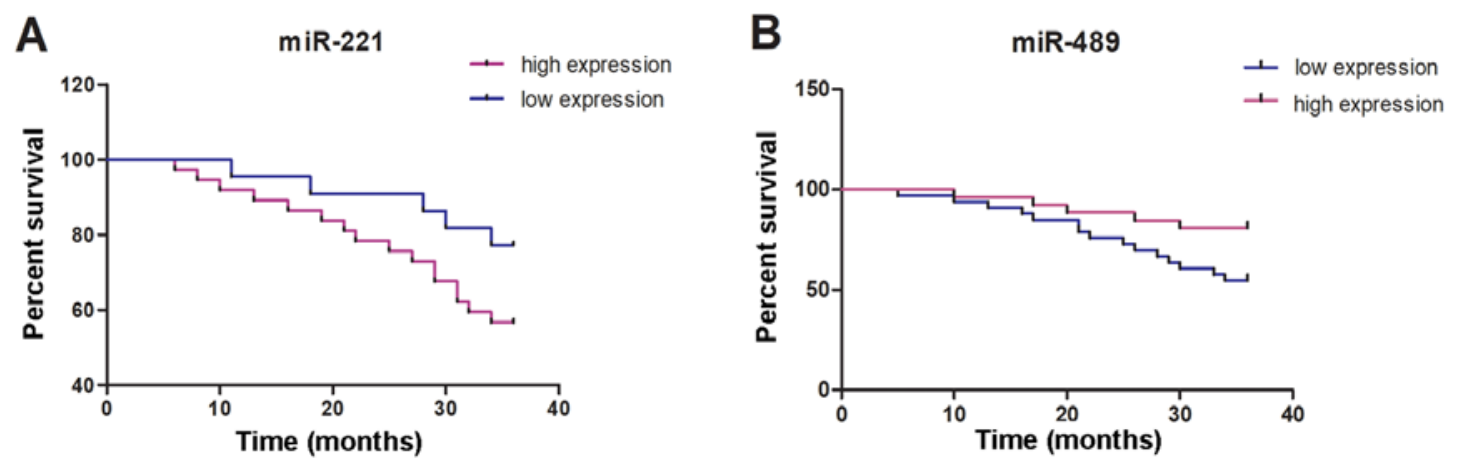

Figure 3. Prognosis and survival of breast cancer patients. Relationship between (A) miR-221 and (B) miR-489 expression levels and the prognosis and survival of patients.

$(\mathrm{P}=0.049)$. The 1-, 2 - and 3-year survival rates in the miR-489 low-expression group were $93.94,75.76$ and $54.55 \%$, respectively, whereas those in the miR-489 high-expression group were $96.15,88.46$ and $80.77 \%$, respectively, which were significantly higher than those in the miR-489 low-expression group $(\mathrm{P}=0.035)$ (Fig. 3).

Relationship between the miR-221 and miR-489 expression levels and the clinicopathological characteristics of the patient in the $R G$. The expression levels of miR-221 and miR-489 in the RG were related to age, family history of breast cancer, type of carcinoma, degree of differentiation, pathological staging and distant metastasis $(\mathrm{P}<0.05)$. Details are presented in Tables III and IV.

\section{Discussion}

Breast cancer is one of the most common malignant tumors among women worldwide. Drug resistance and distant organ metastasis are the main causes of most breast cancer-related deaths (12). In recent years, the mortality of breast cancer has decreased due to the promotion of disease screening; however, the mortality rate remains high $(13,14)$. Early breast cancer is usually treated by adjuvant systemic therapy, including chemotherapy, radiotherapy and endocrine therapy (15). However, due to the toxic effects of these treatment methods, the benefits for the breast cancer patients are not obvious (16). At present, as the pathogenesis of breast cancer has not been completely elucidated and there is no effective reference index 
Table III. Relationship between miR-221 expression level and the clinicopathological characteristics of patients in the RG.

\begin{tabular}{|c|c|c|c|c|}
\hline Clinicopathological characteristics & $\mathrm{n}$ & Expression level & $\mathrm{t}$ & P-value \\
\hline Age (years) & & & 2.119 & 0.038 \\
\hline$\geq 40$ & 28 & $7.33 \pm 2.12$ & & \\
\hline$<40$ & 34 & $6.41 \pm 1.26$ & & \\
\hline Family history of breast cancer & & & 2.379 & 0.021 \\
\hline Yes & 21 & $7.62 \pm 2.47$ & & \\
\hline No & 41 & $6.53 \pm 1.15$ & & \\
\hline Type of carcinoma & & & 2.163 & 0.035 \\
\hline Infiltrating carcinoma & 49 & $7.59 \pm 1.31$ & & \\
\hline Non-infiltrating carcinoma & 13 & $6.73 \pm 1.12$ & & \\
\hline Degree of differentiation & & & 2.284 & 0.026 \\
\hline Moderately and poorly differentiated & 50 & $7.63 \pm 1.25$ & & \\
\hline Highly differentiated & 12 & $6.72 \pm 1.19$ & & \\
\hline Pathological staging & & & 2.056 & 0.044 \\
\hline Stages I-II & 27 & $7.09 \pm 1.26$ & & \\
\hline Stages III-IV & 35 & $7.88 \pm 1.66$ & & \\
\hline Distant metastasis & & & 2.066 & 0.043 \\
\hline Yes & 25 & $7.89 \pm 1.86$ & & \\
\hline No & 37 & $7.02 \pm 1.45$ & & \\
\hline
\end{tabular}

RG, research group.

Table IV. Relationship between miR-489 expression level and the clinicopathological characteristics of patients in the RG.

\begin{tabular}{|c|c|c|c|c|}
\hline Clinicopathological characteristics & $\mathrm{n}$ & Expression level & $\mathrm{t}$ & P-value \\
\hline Age (years) & & & 2.186 & 0.033 \\
\hline$\geq 40$ & 28 & $0.82 \pm 0.06$ & & \\
\hline$<40$ & 34 & $0.86 \pm 0.08$ & & \\
\hline Family history of breast cancer & & & 2.236 & 0.029 \\
\hline Yes & 21 & $0.80 \pm 0.05$ & & \\
\hline No & 41 & $0.83 \pm 0.05$ & & \\
\hline Type of carcinoma & & & 2.458 & 0.017 \\
\hline Infiltrating carcinoma & 49 & $0.78 \pm 0.05$ & & \\
\hline Non-infiltrating carcinoma & 13 & $0.82 \pm 0.06$ & & \\
\hline Degree of differentiation & & & 4.362 & $<0.001$ \\
\hline Moderately and poorly differentiated & 50 & $0.78 \pm 0.05$ & & \\
\hline Highly differentiated & 12 & $0.83 \pm 0.04$ & & \\
\hline Pathological staging & & & 2.240 & 0.029 \\
\hline Stages I-II & 27 & $0.86 \pm 0.06$ & & \\
\hline Stages III-IV & 35 & $0.83 \pm 0.04$ & & \\
\hline Distant metastasis & & & 2.278 & 0.026 \\
\hline Yes & 25 & $0.86 \pm 0.07$ & & \\
\hline No & 37 & $0.91 \pm 0.06$ & & \\
\hline
\end{tabular}

RG, research group.

for the diagnosis and prognosis, biological indicators and new targets for the diagnosis and treatment of breast cancer have become the research focus in clinical practice (17). In recent years, a number of studies have been reported on miRNA as 
a new indicator for cancer diagnosis and treatment (18-20). miRNA is a small non-coding region of 20-22 nucleotide RNA. Under normal circumstances, miRNAs play a role in feedback mechanisms by protecting proliferation, differentiation, apoptosis and other processes of cells, and are key links in a number of biological processes $(21,22)$. Previous studies have proven that miRNAs can act as oncogenes or tumor suppressor genes according to the functions of their target genes (23). Among them, some studies have confirmed that miR-221 stimulates different types of tumors and downregulates some tumor suppressor genes. Upregulation of miR-221 is linked to the occurrence of various hematological diseases and solid malignant tumors (24). However, miR-489 can play a part in proliferation, survival and invasion of cells by regulating genes (25). With the deepening of research, it is gradually considered that miR-221 and miR-489 may be closely related to the occurrence and development of breast cancer $(26,27)$.

The results of the present study revealed that the expression level of miR-221 in patients of the RG was significantly higher than that in the CG, and the expression level of miR-489 in the RG was significantly lower than that in the CG, suggesting that miR-221 and miR-489 might participate in the occurrence and development of breast cancer, in agreement with the research results of Patel et al, Pan et al and Ye et al (28-30). ROC curve analysis showed that when the cut-off value was 6.806 , the sensitivity and specificity of miR-221 in the diagnosis of breast cancer were 61.29 and $85.19 \%$, respectively; when the cut-off value was 0.903 , the sensitivity and specificity of miR-489 were 66.13 and $85.19 \%$, respectively; and when the cut-off value was 0.165 , the combined detection of the two had higher specificity than the single detection of miR-221 and $\mathrm{miR}-489$. The relationship between the expression levels of miR-221 and miR-489 and the clinicopathological characteristics of breast cancer patients was further analyzed. miR-221 and miR-489 were significantly associated with age, family history of breast cancer, pathological type of carsinoma, degree of differentiation, pathological staging and distant metastasis, suggesting that miR-221 and miR-489 are closely associated to differentiation and proliferation of breast cancer cells. The prognosis of the miR-221 and miR-489 high- and low-expression groups were compared for 3 years, respectively. The survival rate of the miR-221 high-expression group was significantly lower than that of the miR-221 low-expression group, and the survival rate of the miR-489 low-expression group was significantly lower than that of the miR-489 high-expression group, suggesting that miR-221 and miR-489 are relevant to the prognosis of breast cancer patients. The aim of improving prognosis could be achieved by detecting the expression levels of miR-221 and miR-489 in breast cancer patients.

At present, biopsy is still the only definite way to diagnose breast cancer, although it causes great damage to the body. Therefore, a convenient and effective biomarker is urgently needed in clinic for the diagnosis and treatment of breast cancer diseases. As miRNA can be detected from serum, plasma, body fluids and tissues, it has become a hot spot in the research of tumor diseases. In the present study, the expression levels of miR-221 and miR-489 in the blood of breast cancer patients were analyzed, and it was shown that miR-221 and
miR-489 can be effective indicators for the diagnosis and treatment of breast cancer diseases. However, there are still some limitations in this study, such as the small number of experimental subjects, the short follow-up time, and the lack of basic experiments. The mechanism of miR-221 and miR-489 on breast cancer is not yet clear. Further research is needed to continuously improve our experiments in order to obtain the best and most accurate experimental results.

In conclusion, miR-221 expression is upregulated and miR-489 expression is downregulated in the blood of breast cancer patients, which has a certain impact on prognosis. In the future, miR-221 can be used as an effective indicator for the diagnosis, treatment and prognosis of breast cancer.

\section{Acknowledgements}

Not applicable.

\section{Funding}

No funding was received.

\section{Availability of data and materials}

The datasets used and/or analyzed during the current study are available from the corresponding author on reasonable request.

\section{Authors' contributions}

FL conceived and designed the study, performed the experiments and the statistical analysis, and wrote the manuscript. The author read and approved the final version of the manuscript.

\section{Ethics approval and consent to participate}

The study was approved by the Ethics Committee of the First Teaching Hospital of Tianjin University of Traditional Chinese Medicine (Tianjin, China). All subjects who participated in this research had complete clinical data. Signed written informed consents were obtained from the participants and/or their guardians.

\section{Patient consent for publication}

Not applicable.

\section{Competing interests}

The authors declare that they have no competing interests.

\section{References}

1. Ghislain I, Zikos E, Coens C, Quinten C, Balta V, Tryfonidis K, Piccart M, Zardavas D, Nagele E, Bjelic-Radisic V, et al; European Organisation for Research and Treatment of Cancer (EORTC) Quality of Life Group; Breast Cancer Group; EORTC Headquarters: Health-related quality of life in locally advanced and metastatic breast cancer: Methodological and clinical issues in randomised controlled trials. Lancet Oncol 17: e294-e304, 2016.

2. DeSantis CE, Ma J, Goding Sauer A, Newman LA and Jemal A: Breast cancer statistics, 2017, racial disparity in mortality by state. CA Cancer J Clin 67: 439-448, 2017. 
3. Malvezzi M, Carioli G, Bertuccio P, Boffetta P, Levi F, La Vecchia $C$ and Negri E: European cancer mortality predictions for the year 2019 with focus on breast cancer. Ann Oncol 30: 781-787, 2019.

4. Gao C, Li H, Zhuang J, Zhang H, Wang K, Yang J, Liu C, Liu L, Zhou $C$ and Sun C: The construction and analysis of ceRNA networks in invasive breast cancer: A study based on The Cancer Genome Atlas. Cancer Manag Res 11: 1-11, 2018.

5. Meng X, Müller V, Milde-Langosch K, Trillsch F, Pantel K and Schwarzenbach H: Diagnostic and prognostic relevance of circulating exosomal miR-373, miR-200a, miR-200b and miR-200c in patients with epithelial ovarian cancer. Oncotarget 7: 16923-16935, 2016.

6. Yu Y, Nangia-Makker P, Farhana L, G Rajendra S, Levi E and Majumdar AP: miR-21 and miR-145 cooperation in regulation of colon cancer stem cells. Mol Cancer 14: 98, 2015.

7. Ying $X, W u$ Q, Wu X, Zhu Q, Wang X, Jiang L, Chen X and Wang X: Epithelial ovarian cancer-secreted exosomal miR-222-3p induces polarization of tumor-associated macrophages. Oncotarget 7: 43076-43087, 2016.

8. Mukohyama J, Shimono Y, Dalerba P, Isobe T, Hu Q, Sahoo D, Shibuya N, Minami H, Mimori K, Kakeji Y, et al: Epigenetic regulation of colorectal cancer stem cells by the miR-221/QKI5 axis. Cancer Res 78: 488, 2018.

9. Fang Z, Zhong M, Wang Y, Yuan X, Guo H, Yao Y, Feng M, Chen J, Xiong J and Xiang X: miR-381 and miR-489 suppress cell proliferation and invasion by targeting CUL4B via the $\mathrm{Wnt} / \beta$-catenin pathway in gastric cancer. Int J Oncol 54: 733-743, 2019.

10. Xu D, Liu R, Meng L, Zhang Y, Lu G and Ma P: Long non-coding RNA ENST01108 promotes carcinogenesis of glioma by acting as a molecular sponge to modulate miR-489. Biomed Pharmacother 100: 20-28, 2018.

11. Vergara-Ortega DN, Sevilla-Reyes EE, Herrera-Ortiz A, Torres-Ibarra L, Salmerón J, Lazcano-Ponce E and SánchezAlemán MA: Real time PCR to evaluate HSV-2 shedding from anal and genital samples among men who have sex with men, living with HIV. J Med Virol 90: 745-752, 2018.

12. Singh SK, Singh S, Lillard JW Jr and Singh R: Drug delivery approaches for breast cancer. Int J Nanomedicine 12: 6205-6218, 2017.

13. Welch HG, Prorok PC, O'Malley AJ and Kramer BS: Breast-cancer tumor size, overdiagnosis, and mammography screening effectiveness. N Engl J Med 375: 1438-1447, 2016.

14. Burwinkel B, Cuk K, Zucknick M and Madhavan D: Circulating miRNAs as markers for breast cancer. US Patent 10,316,367. Filed June 21, 2013; issued June 11, 2019.

15. Sparano JA, Gray RJ, Makower DF, Pritchard KI, Albain KS, Hayes DF, Geyer CE Jr, Dees EC, Goetz MP, Olson JA Jr, et al: Adjuvant chemotherapy guided by a 21-gene expression assay in breast cancer. N Engl J Med 379: 111-121, 2018.

16. Cardoso F, van't Veer LJ, Bogaerts J, Slaets L, Viale G, Delaloge S, Pierga JY, Brain E, Causeret S, DeLorenzi M, et al; MINDACT Investigators: 70-gene signature as an aid to treatment decisions in early-stage breast cancer. N Engl J Med 375: 717-729, 2016.
17. Malih S, Saidijam M and Malih N: A brief review on long noncoding RNAs: A new paradigm in breast cancer pathogenesis, diagnosis and therapy. Tumour Biol 37: 1479-1485, 2016.

18. Shah MY, Ferrajoli A, Sood AK, Lopez-Berestein G and Calin GA: MicroRNA therapeutics in cancer - an emerging concept. EBioMedicine 12: 34-42, 2016.

19. Rupaimoole R and Slack FJ: MicroRNA therapeutics: Towards a new era for the management of cancer and other diseases. Nat Rev Drug Discov 16: 203-222, 2017.

20. Acunzo M, Romano G, Wernicke D and Croce CM: MicroRNA and cancer - a brief overview. Adv Biol Regul 57: 1-9, 2015.

21. Reddy KB: MicroRNA (miRNA) in cancer. Cancer Cell Int 15: $38,2015$.

22. Cheng CJ, Bahal R, Babar IA, Pincus Z, Barrera F, Liu C, Svoronos A, Braddock DT, Glazer PM, Engelman DM, et al: MicroRNA silencing for cancer therapy targeted to the tumour microenvironment. Nature 518: 107-110, 2015.

23. Lou W, Liu J, Gao Y, Zhong G, Chen D, Shen J, Bao C, Xu L, Pan J, Cheng J, et al: MicroRNAs in cancer metastasis and angiogenesis. Oncotarget 8: 115787-115802, 2017.

24. Santolla MF, Lappano R, Cirillo F, Rigiracciolo DC, Sebastiani A, Abonante S, Tassone P, Tagliaferri P, Di Martino MT, Maggiolini M, et al: miR-221 stimulates breast cancer cells and cancer-associated fibroblasts (CAFs) through selective interference with the A20/c-Rel/CTGF signaling. J Exp Clin Cancer Res 37: 94, 2018.

25. Gao S, Liu H, Hou S, Wu L, Yang Z, Shen J, Zhou L, Zheng SS and Jiang B: miR-489 suppresses tumor growth and invasion by targeting HDAC7 in colorectal cancer. Clin Transl Oncol 20: 703-712, 2018

26. Chen X, Wang YW, Xing AY, Xiang S, Shi DB, Liu L, Li YX and Gao P: Suppression of SPIN1-mediated PI3K-Akt pathway by miR-489 increases chemosensitivity in breast cancer. J Pathol 239: 459-472, 2016.

27. Roscigno G, Quintavalle C, Donnarumma E, Puoti I, Diaz-Lagares A, Iaboni M, Fiore D, Russo V, Todaro M, Romano G, et al: miR-221 promotes stemness of breast cancer cells by targeting DNMT3b. Oncotarget 7: 580-592, 2016.

28. Patel Y, Shah N, Lee JS, Markoutsa E, Jie C, Liu S, Botbyl R, Reisman D, Xu P and Chen $\mathrm{H}$ : A novel double-negative feedback loop between miR-489 and the HER2-SHP2-MAPK signaling axis regulates breast cancer cell proliferation and tumor growth. Oncotarget 7: 18295-18308, 2016.

29. Pan Y, Li J, Zhang Y, Wang N, Liang H, Liu Y, Zhang CY, Zen K and $\mathrm{Gu} \mathrm{H}$ : Slug-upregulated miR-221 promotes breast cancer progression through suppressing E-cadherin expression. Sci Rep 6: 25798, 2016.

30. Ye Z, Hao R, Cai Y, Wang X and Huang G: Knockdown of miR-221 promotes the cisplatin-inducing apoptosis by targeting the BIM-Bax/Bak axis in breast cancer. Tumour Biol 37: 4509-4515, 2016

This work is licensed under a Creative Commons Attribution-NonCommercial-NoDerivatives 4.0 International (CC BY-NC-ND 4.0) License. 